\title{
Promoting Intersystem Crossing of Fluorescent Molecule via Single Functional Group Modification
}

\author{
Ran Liu, ${ }^{a}$ Xing Gao, ${ }^{\mathrm{b}}$ Mario Barbatti, ${ }^{{ }^{*} \mathrm{c}}$ Jun Jiang, ${ }^{\mathrm{a}}$ Guozhen Zhang ${ }^{{ }^{\mathrm{a}}}$ \\ ${ }^{\text {a }}$ Hefei National Laboratory for Physical Sciences at the Microscale, iChEM (Collaborative \\ Innovation Center of Chemistry for Energy Materials), School of Chemistry and Materials \\ Science, University of Science and Technology of China, Hefei, Anhui 230026, P. R. China. \\ E-mail: guozhen@ustc.edu.cn. \\ ${ }^{\mathrm{b}}$ Department of Chemistry, $930 \mathrm{~N}$ University Ave, University of Michigan, Ann Arbor, MI \\ 48109, USA \\ c Aix Marseille Univ, CNRS, ICR, Marseille, France. E-mail: mario.barbatti@univ-amu.fr \\ Website: www.barbatti.org
}

\begin{abstract}
Pure light-atoms organic phosphorescent molecules have been under scientific scrutiny because they are inexpensive, flexible, and environment friendly. The development of such materials, however, faces a bottleneck problem of intrinsically small spin-orbit couplings (SOC), which can be addressed by seeking a proper balance between intersystem crossing (ISC) and fluorescence rates. Using Nsubstituted naphthalimides (NNI) as the prototype molecule, we applied chemical modifications with several electrophilic and nucleophilic functional groups, to approach the goal. The selected electron donating groups actively restrain the fluorescence, enabling an efficient ISC to the triplet manifold. Electron withdrawing groups do not change the luminescent properties of the parent species. The changes in ISC and fluorescence rates are related to the nature of the lowest singlet state, which changes from localized excitation into charge-transfer excitation. This finding opens an alternative strategy for designing pure light-atoms organic phosphorescent molecules for emerging luminescent materials applications.
\end{abstract}


TOC Graphic

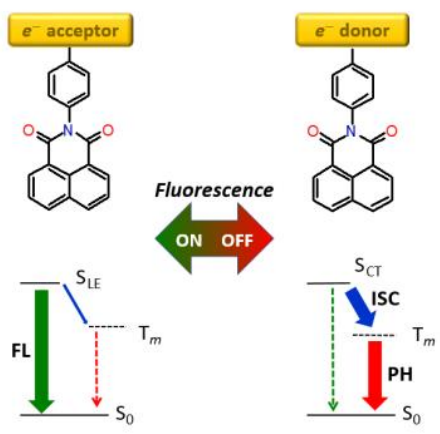

Molecular phosphorescence has a high potential in information processing applications, including sensors, biological imaging, and LEDs, which has promoted a vivid search for efficient, low-cost, and environment-friendly phosphorescent materials. ${ }^{1-6}$ Naturally, a crucial point in this search is to develop molecules with efficient intersystem crossing (ISC), the nonradiative transition between electronic states with different spin multiplicity. ${ }^{7-14}$ In organometallic LEDs, for instance, the interaction between the singlet metal-to-ligand charge transfer $\left({ }^{1} \mathrm{MLCT}\right)$ and the lowest triplet metal-to-ligand charge transfer ( $\left.{ }^{3} \mathrm{MLCT}\right)$ states leads to robust ISC, resulting in high phosphorescence quantum yield. ${ }^{15-16}$

To date, many phosphorescent molecules are either organometallic molecules or organic molecules with halogen atoms. ${ }^{17-18}$ Such dominance has two causes, both well-documented. First, heavy, occasionally magnetic metal ions help to strength the spin-orbit coupling (SOC), which is the root of ISC. ${ }^{19-20}$ Secondly, charge transfer (CT) processes contribute to tuning the relevant excited energies favorably. ${ }^{21-23}$ That is, the poor superposition of the orbitals from the metal center and ligand results in tiny singlet-triplet energy gap ( $\Delta E_{S T}$, the energy difference between the lowest singlet excited state and neighboring triplet state). ${ }^{3}$ Thus, the ISC efficiency can be improved by either reducing $\Delta E_{S T}$ or enlarging the SOC matrix element ${ }^{24-25}$ (see also eq. (1)). 
It has been reported that small $\Delta E_{S T}$ led to efficient ISC and thereby strong phosphorescence in dibenzothiophene-S,S-dioxide and 2-biphenyl-4,6-bis(12phenylindolo[2,3-a]carbazole-11-yl)-1,3,5-triazine complexes. $^{26-27}$ In suitable conditions, the metal-ligand compounds may emit phosphorescence thanks to the ligand-localized nature $\left({ }^{3} \mathrm{IL}^{*}\right.$ or $\left.{ }^{3} \pi-\pi^{*}\right)$ of the lowest triplet state and the relaxation of ${ }^{3} \mathrm{LMCT} /{ }^{3} \mathrm{MLCT}$ to ${ }^{3} \pi \mathrm{LC}^{*} \cdot{ }^{28}$ However, the risk of toxicity and instability of organometallic or halo-organic molecules limits their applications. ${ }^{29}$

By comparison, pure light-atoms organic molecules could be an alternative because of their low cost, potentially low toxicity, and high engineering flexibility. Recently, pure light-atoms organic materials have emerged as a promising type of phosphorescent materials. Their luminescent properties were modified through crystallization and aggregation-induced emission enhancement to reduce nonradiative losses. ${ }^{29-33}$ Nevertheless, the designing of pure light-atoms organic phosphorescent chromophores has still been a significant challenge because of their tiny SOC, ranging from around $1 \mathrm{~cm}^{-1}$ in El-Sayed ${ }^{34}$ forbidden transitions to typically 50 to $100 \mathrm{~cm}^{-1}$ in El-Sayed allowed transitions. ${ }^{35-36}$ Such SOC values are truly too small compared to those above $500 \mathrm{~cm}^{-1}$ observed in organometallic systems, ${ }^{37}$ especially considering that the ISC rate depends on their squared values.

Despite their tiny SOCs, specific chemical modification to pure light-atoms organic fluorescent molecules with functional groups have successfully activated phosphorescence. This is the case, for instance, of N-substituted naphthalimides (NNI), whose luminescent properties can be fundamentally altered when the functional group $-\mathrm{OCH}_{3}$ is added, as reported by Chen et al. ${ }^{3}$ Specifically, NNI is fluorescence active but phosphorescence inactive. By contrast, $\mathrm{NNI}-\mathrm{OCH}_{3}$ is phosphorescence active but fluorescence inactive (see Figure 1(a)). The proposed mechanism ${ }^{3}$ explaining the phosphorescence activation in NNI- $\mathrm{OCH}_{3}$ claims that both the first singlet excited state $\left(\mathrm{S}_{1}\right)$ and the closest triplet excited state of $\mathrm{NNI}-\mathrm{OCH}_{3}$ have charge-transfer (CT) 
characters, reducing $\Delta E_{S T}$ and, consequently, enhancing the ISC rate (see Figure $1(b))$.

(a)
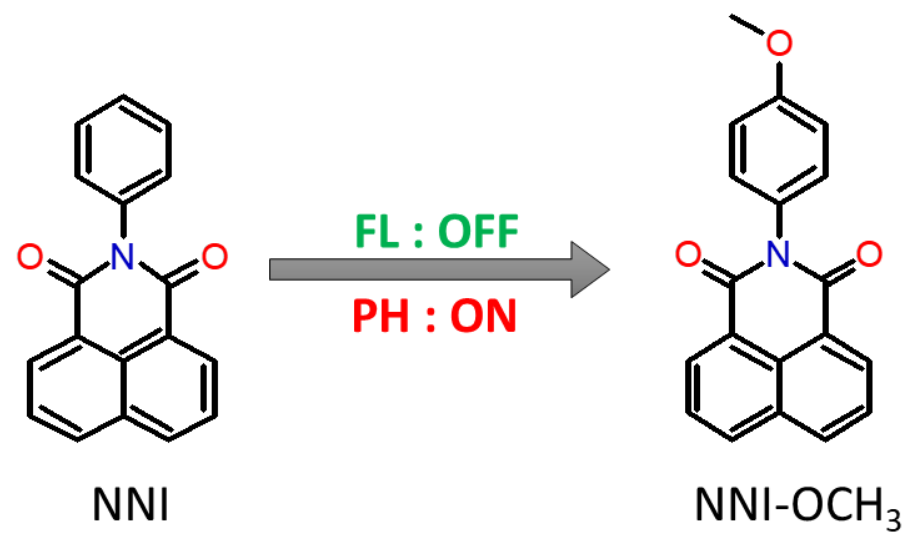

(b) Model 1 (Chen et al. 2016)
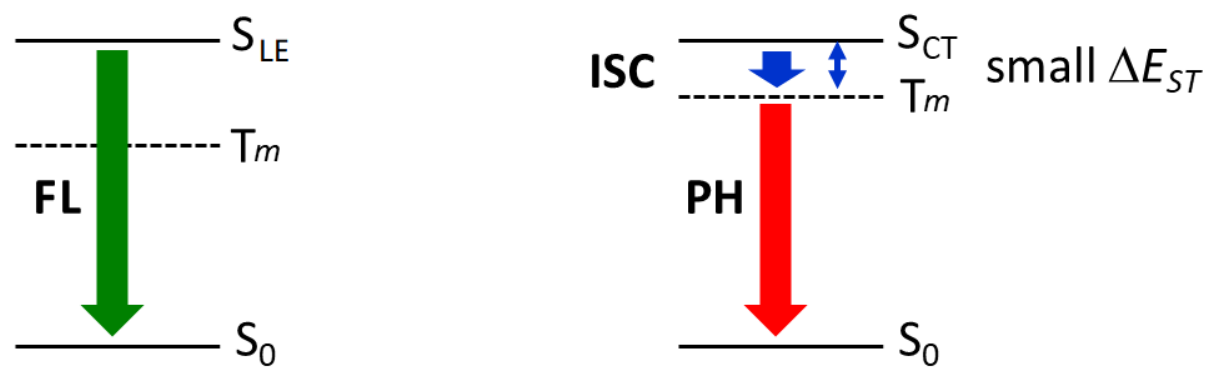

(c) Model 2 (Present work)
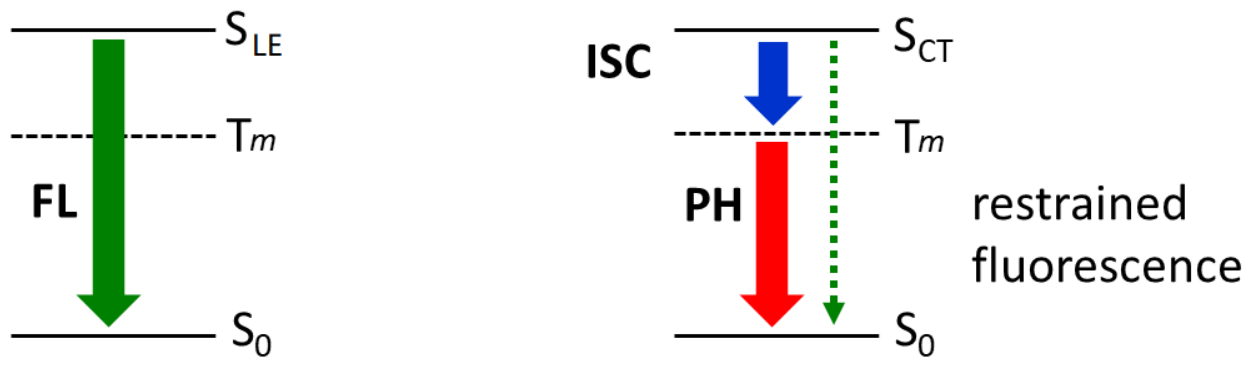

Figure 1. (a) The luminescence properties of NNI and NNI- $\mathrm{OCH}_{3}$. The addition of $-\mathrm{OCH}_{3}$ turns the fluorescent NNI into a phosphorescent molecule. (b) A previously proposed mechanism to explain $\mathrm{NNI}-\mathrm{OCH}_{3}$ phosphorescence is based on a small $\Delta E_{S T}$ due to low-lying CT states. $^{3}$ (c) The mechanism proposed in this work is based on restrained fluorescence also caused by low-lying CT states. 
These findings prompt us to investigate the photophysics of NNI from a theoretical standpoint, to learn how to control the luminescence behavior of a dye molecule through a delicate modification of its chemical structure. Here, we applied density functional theory (DFT) and time-dependent DFT (TD-DFT) to map electronhole distributions and to estimate fluorescence and phosphorescence rates in NNI and NNI substituted with diverse functional groups. By studying functional groups that are either electron withdrawing (EWGs: $-\mathrm{F}$ and $-\mathrm{COOH}$ ) or electron donating (EDGs: $\mathrm{OH}$ and $-\mathrm{OCH}_{3}$ ), we aimed to understand how these different classes of functional substitutions impact the luminescent properties.

We have found out that EDG substitutions strongly enhance the triplet population, while EWG substitutions have little effect compared to the parent system. As proposed by Chen et al., ${ }^{3}$ the CT character of the $\mathrm{S}_{1}$ state in NNI-EDG is the crucial piece to understand the enhanced ISC in these systems. Nevertheless, the reason underlying the ISC enhancement is not a reduction of $\Delta E_{S T}$, but rather a stronglyrestrained fluorescence due to transition dipole moment reduction (Figure 1(c)), which compensates for the small SOCs.

Based on the semiclassical Marcus theory, the ISC rate, $k_{I S C}=\tau_{I S C}^{-1}$, from $\mathrm{S}_{1}$ to a neighbor triplet state $\mathrm{T}_{m}$ can be estimated as ${ }^{38}$

$$
k_{I S C}=\frac{2 \pi}{\hbar}\left|V_{S O C}\right|^{2} \frac{1}{\sqrt{4 \pi \lambda k_{B} T}} \exp \left(-\frac{\left(\Delta G_{S T}^{v}\right)^{2}}{4 \lambda k_{B} T}\right)
$$

where $V_{S O C}$ is the spin-orbit coupling (SOC) matrix element, $\lambda$ is the reorganization energy, and $\Delta G_{S T}^{v}$ is the vertical free Gibbs energy variation. As usual, $\hbar$ is the reduced Planck constant, $k_{B}$ is the Boltzmann constant, and $T$ is the absolute temperature. The reorganization energy was approximated as $\lambda \approx E_{T_{2}}\left(\mathbf{R}_{\min S_{1}}\right)-E_{T_{2}}\left(\mathbf{R}_{\min T_{2}}\right)$ and the vertical free energy gap as 
$\Delta G_{S T}^{v} \approx \Delta E_{S T}=E_{T_{m}}\left(\mathbf{R}_{\min S_{1}}\right)-E_{S_{1}}\left(\mathbf{R}_{\min S_{1}}\right)$. The SOC matrix elements of selected singlet-triplet channels were calculated at the optimized structure of $S_{1}$. They were evaluated adopting the Breit-Pauli (BP) spin-orbital Hamiltonian with effective-charge approximation, ${ }^{39}$ using Casida's wavefunction Ansatz. ${ }^{40}$ Given proper approximations (See details in the Supporting Information), the simplified rate model in Eq. (1) is supposed to deliver a qualitative estimate of the ISC rate. This limitation is not a major impediment for us, as the rates here are only needed for a qualitative comparison between different photophysical processes.

Meantime, the fluorescence rate, $k_{F L}=\tau_{F L}^{-1}$, was estimated as ${ }^{41}$

$$
k_{F L}=\frac{e^{2}}{2 \pi \varepsilon_{0} m_{e} c^{3} \hbar^{2}} \Delta E^{2} f
$$

where $\varepsilon_{0}$ is the vacuum permittivity, $m_{e}$ is the electron mass, $e$ is the electron charge, $c$ is the speed of light. $\Delta E=E_{S_{0}}\left(\mathbf{R}_{\min S_{1}}\right)-E_{S_{1}}\left(\mathbf{R}_{\min S_{1}}\right)$ and $f$ are the $S_{1}-S_{0}$ energy gap and the oscillator strength calculated at the $S_{1}$ minimum.

The chemical structures of NNI and its four derivatives with single parasubstitution are collected in the upper half of Figure 2. We first examined the ground states of all species. Intriguingly, regardless of the substituent added to NNI, the benzene and naphthalimide groups are always perpendicular to each other in optimized structures (See Cartesian coordinates in Supporting Information). Further, vertical excitation calculations based on optimized $S_{0}$ geometries show that the $S_{1}$ states of all species are almost the same (See Table S1 in Supporting Information). Thus, for NNI$\mathrm{OCH}_{3}$ and NNI-OH, there may be some configurational change during the relaxation of $S_{1}$ that can induce the change of its nature.

The electron-hole distributions of their respective $S_{1}$ and $T_{2}$ states based on optimized $S_{1}$ structures are collected in the lower half of Figure 2. Compared to the NNI molecule, the EDGs concerned in the work $\left(-\mathrm{OH}\right.$ and $\left.-\mathrm{OCH}_{3}\right)$ alter the nature of 
$\mathrm{S}_{1}$ and $\mathrm{T}_{2}$ from locally excited states to charge-transfer (CT) states, with an electron transfer from the benzene to the naphtalimide group. Noticeably, this shift is closely related to the conformational change of $S_{1}$ and $T_{2}$. For NNI, the benzene and the naphthalimide groups are perpendicular to each other, while for NNI-X $(\mathrm{X}=-\mathrm{OH}$ or $\mathrm{OCH}_{3}$ ), they are not. Also, the $\mathrm{S}_{1}$ of NNI-OH and NNI- $\mathrm{OCH}_{3}$ is a dark state because the two molecular orbitals involved in $\mathrm{S}_{1}$ (see Table $\mathrm{S} 2$ in the Supporting Information) are poorly $\pi$-conjugated as the two corresponding molecular fragments appreciably deviated from co-planarity. ${ }^{42}$ Meanwhile, the EWGs groups $-\mathrm{F}$ and $-\mathrm{COOH}$ left almost unchanged the nature of $S_{1}$ and $T_{2}$.

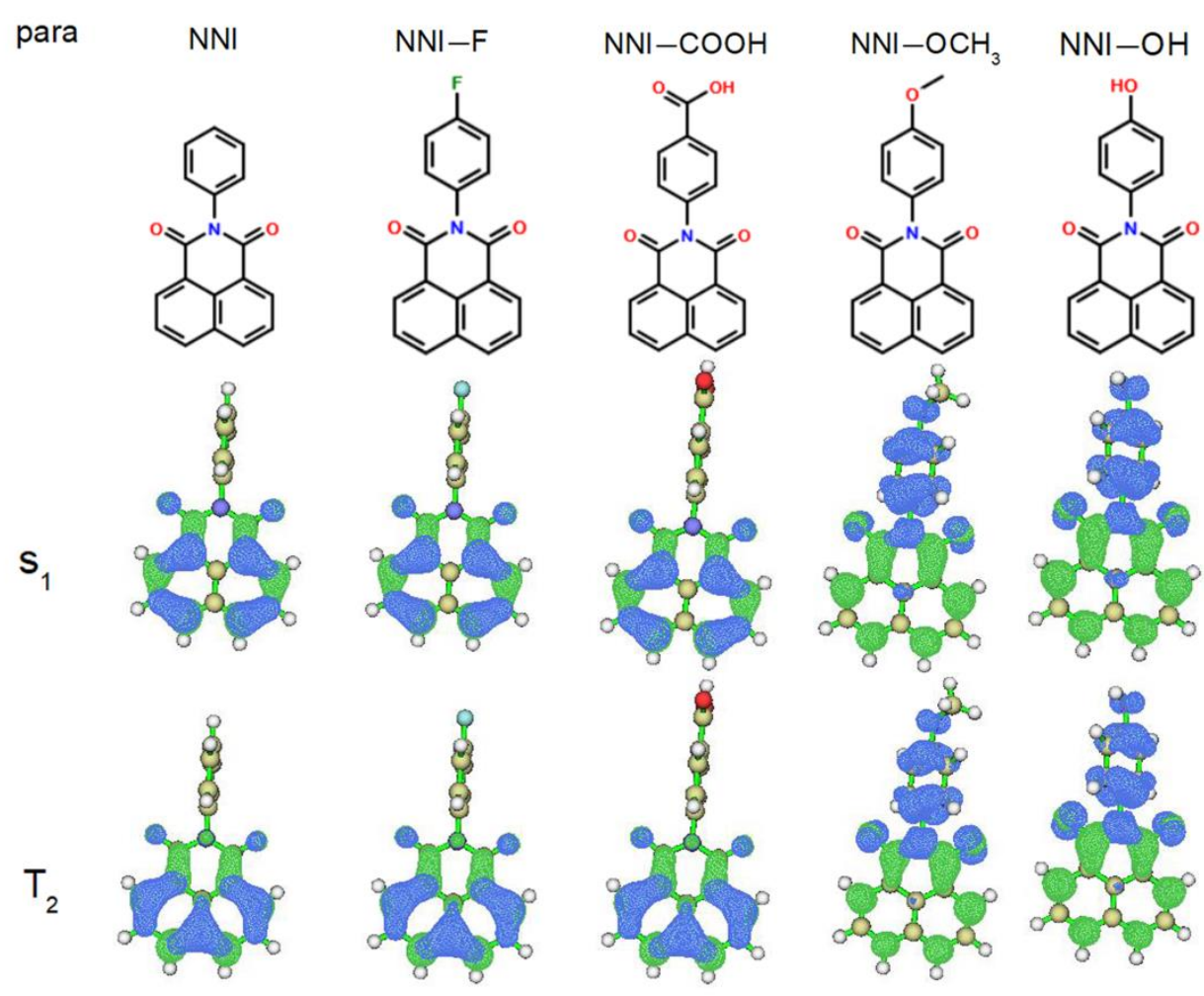

Figure 2. Upper half: chemical structures; lower half: the electron-hole distribution of $\mathrm{S}_{1}$ and $\mathrm{T}_{2}$ of NNI and its para-substituted derivatives. (The blue and green regions represent the hole and electron, respectively.) 
The occurrence of an $S_{1}$ state with $\mathrm{CT}$ character in NNI-EDG has major consequences for the photophysics of these molecules. While the $S_{1} \rightarrow T_{2}$ ISC rate in the EWG substitutions is not significantly altered in relation to the parent NNI (Table 1), in the case of the EDGs, it shows an increase of three times for $-\mathrm{OCH}_{3}$ and doubling for $-\mathrm{OH}$. Thus, the ISC lifetime shortens from $23-26 \mathrm{~ns}$ in NNI and NNI-EWG to about 8 - 11 ns in NNI-EDG. The data in Table 1 also show that the ISC rate variation is caused by the increase of the $V_{S O C}$, from 0.5 to $1.3 \mathrm{~cm}^{-1}$ upon EDG substitution, which compensates the small but unfavorable growth of $\Delta E_{S T}$.

Curiously, the reason $V_{S O C}$ is so small in either NNI-EWGs or NNI-EDGs is not the same. In NNI-EWGs (and in the parent NNI), the singlet-triplet transition between $\pi \pi^{*}$ states is unfavorable by the El-Sayed rule. ${ }^{34}$ In NNI-EDGs, $V_{S O C}$ is tiny due to the local nature of the spin-orbit coupling Hamiltonian, ${ }^{43}$ which tends to return vanishing elements for non-local transitions, as in CT states.

Table $1 . S_{1}-T_{2}$ energy gap $\left(\Delta E_{S T}\right)$ at the $S_{1}$ minimum, reorganization energy $(\lambda)$, and SOC ( $\left.V_{S O C}\right)$. ISC rate $\left(k_{I S C}\right)$, ISC rate relative to that of NNI $\left(k_{r e l}\right)$, ISC lifetime $\left(\tau_{I S C}\right)$. All quantities calculated for NNI and its para- and meta-substituted derivatives with electron withdrawing $(-\mathrm{F}$ and $-\mathrm{COOH})$ and electron donating $\left(-\mathrm{OCH}_{3}\right.$ and $\left.-\mathrm{OH}\right)$ groups.

\begin{tabular}{|c|c|c|c|c|c|c|}
\hline & $\Delta E_{S T}(\mathrm{eV})$ & $\lambda(\mathrm{eV})$ & $V_{S O C}\left(\mathrm{~cm}^{-1}\right)$ & $k_{I S C}\left(10^{7} \mathrm{~s}^{-1}\right)$ & $k_{r e l}$ & $\tau_{I S C}(\mathrm{~ns})$ \\
\hline NNI & -0.162 & 0.202 & 0.5 & 4.3 & 1 & 23 \\
\hline \multicolumn{7}{|l|}{ Para } \\
\hline$-\mathrm{F}$ & -0.166 & 0.202 & 0.5 & 4.1 & 1 & 25 \\
\hline$-\mathrm{COOH}$ & -0.170 & 0.202 & 0.5 & 3.9 & 1 & 26 \\
\hline$-\mathrm{OCH}_{3}$ & -0.258 & 0.371 & 1.3 & 12.2 & 3 & 8 \\
\hline$-\mathrm{OH}$ & -0.265 & 0.320 & 1.3 & 8.8 & 2 & 11 \\
\hline \multicolumn{7}{|l|}{ Meta } \\
\hline$-\mathrm{F}$ & -0.168 & 0.202 & 0.5 & 3.9 & 1 & 25.3 \\
\hline$-\mathrm{COOH}$ & -0.169 & 0.202 & 0.5 & 3.9 & 1 & 25.5 \\
\hline$-\mathrm{OCH}_{3}$ & -0.268 & 0.368 & 2.2 & 31.0 & 7 & 3.2 \\
\hline$-\mathrm{OH}$ & -0.286 & 0.406 & 2.2 & 27.7 & 6 & 3.6 \\
\hline
\end{tabular}

Such modest changes in the ISC rates would not alone explain the fluorescence switchon reported in Chen et al.'s work. ${ }^{3}$ Nevertheless, the effect of the EDG substitutions on 
the fluorescence is more impressive. In NNI and NNI-EWGs, the fluorescence rate is about $2 \times 10^{8} \mathrm{~s}^{-1}$ (Table 2). In NNI-EDGs, this value is strongly reduced by a factor $\sim 35$. Thus, the fluorescence lifetime is elongated from $5 \mathrm{~ns}$ in NNI and NNI-EWGs to about $180 \mathrm{~ns}$ in NNI-EDGs. This substantial shift in the fluorescence rates caused by the EDG substitutions is a direct result of the CT character of the $S_{1}$ state, which reduces the $S_{1-}$ $S_{0}$ oscillator strength. In addition, large $S_{1}-S_{0}$ energy gaps at the $S_{1}$ minimum (Table 2) suggests that nonradiative decay of $S_{1}$ are not significant for these molecules, even though we have not explicitly investigated nonradiative decay pathways in this work.

Table 2. $\mathrm{S}_{1}-\mathrm{S}_{0}$ energy gap $\Delta E$ and oscillator strength $f$ at the $\mathrm{S}_{1}$ minimum. Fluorescence rate $\left(k_{F L}\right)$, fluorescence rate relative to that of $\mathrm{NNI}\left(k_{r e l}\right)$, and fluorescence lifetime $\left(\tau_{F L}\right)$. All quantities calculated for NNI and its para- and meta-substituted derivatives with electron withdrawing $(-\mathrm{F}$ and $-\mathrm{COOH})$ and electron donating $\left(-\mathrm{OCH}_{3}\right.$ and $\left.-\mathrm{OH}\right)$ groups.

\begin{tabular}{lccccc}
\hline & $\Delta E(\mathrm{eV})$ & $f$ & $k_{F L}\left(10^{8} \mathrm{~s}^{-1}\right)$ & $k_{\text {rel }}$ & $\tau_{F L}(\mathrm{~ns})$ \\
\hline NNI & -3.444 & 0.367 & 1.89 & 1.00 & 5 \\
\hline Para & & & & & \\
\hline$-\mathrm{F}$ & -3.444 & 0.367 & 1.89 & 1.00 & 5 \\
$-\mathrm{COOH}$ & -3.442 & 0.389 & 2.00 & 1.06 & 5 \\
$-\mathrm{OCH}$ & -3.306 & 0.011 & 0.05 & 0.03 & 185 \\
$-\mathrm{OH}$ & -3.340 & 0.012 & 0.06 & 0.03 & 178 \\
\hline Meta & & & & & \\
\hline$-\mathrm{F}$ & -3.444 & 0.368 & 1.89 & 1.00 & 5 \\
$-\mathrm{COOH}$ & -3.443 & 0.378 & 1.90 & 1.03 & 5 \\
$-\mathrm{OCH}$ & -3.049 & 0.007 & 0.03 & 0.02 & 340 \\
$-\mathrm{OH}$ & -3.042 & 0.006 & 0.02 & 0.01 & 408 \\
\hline
\end{tabular}

In short, we show that substitution of selected EDGs in NNI restts in the enhancement of ISC rate between its lowest singlet excited states and adjacent triplet excited states thanks to a larger SOC induces discernable configuration change of the lowest singlet excited state, resulting in a larger SOC between $S_{1}$ and adjacent $T_{2}$ and ensuing enhancement of ISC rate. In contrast to the hypothesis in the experimental work which emphasizes the decisive role of reducing singlet-triplet gap to boost ISC, we 
show that it follows an alternative mechanism for NNI species. Moreover, the dark nature of the $S_{1}$ state in the NNI-EDG, which also roots from the structural change of $\mathrm{S}_{1}$, strongly elongates the fluorescence lifetime, making it slower than ISC and favoring the phosphorescence in these species. Thus, our theoretical results suggest the significance of configuration tuning of chromophores on their luminescent properties, casting new physical insight into the hypothesis proposed in the precedent work of Chen et al. ${ }^{3}$

To further exploit this design strategy, we studied the effect of substitutions in the meta- position. Our results, presented below, revealed the general relationship between the mechanism of intersystem crossing and the charge-transfer excited-state process.

Figure 3 shows the chemical structures of NNI and its four derivatives with single meta-substitution, and the electron-hole distributions of their $\mathrm{S}_{1}$ and $\mathrm{T}_{2}$. They are like their counterpart species of para-substitution in both geometric and electronic structures.

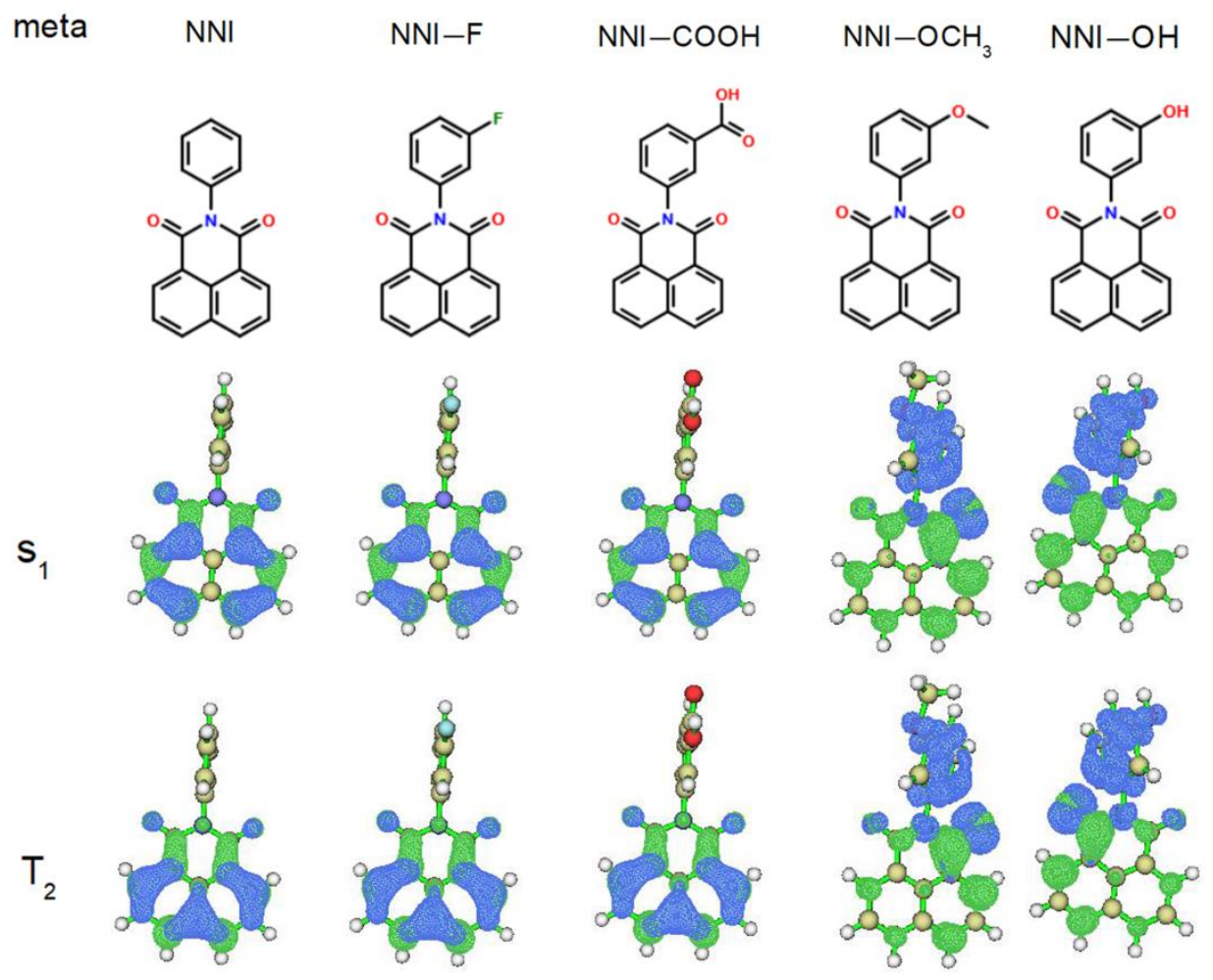


Figure 3. Upper half: chemical structures; lower half: the electron-hole distribution of $\mathrm{S}_{1}$ and $\mathrm{T}_{2}$ of NNI and its meta-substitution derivatives.

The $\mathrm{S}_{1}-\mathrm{T}_{2}$ energy gaps of each meta-substituted species are almost invariant compared to their para-substituted counterpart, as seen from the data in Table 1 and Table 2. The natures of $S_{1}$ are barely changed as well. (See Table S2 and Table S3 in the Supporting Information). The main reason for the insensitivity to the substitution position on the excitation energy of $S_{1}$ lies in that the region the electron moves out during the $\mathrm{S}_{0} \rightarrow \mathrm{S}_{1}$ excitation does not directly involve the added group.

The effects of the substitutions on the ISC and fluorescence rates are also qualitatively the same as those observed in the para-substitutions. Nevertheless, there are some interesting quantitative variations.

The ISC rates of the meta-NNI-EDGs are about 6 to 7 times larger than that of NNI (Table 1). In the para-NNI-EDGs, this growth was no larger than 3 . The reason for this additional enhancement is once again in $V_{S O C}$, which reaches $2.2 \mathrm{~cm}^{-1}$ in metaNNI-EDGs against $0.5 \mathrm{~cm}^{-1}$ in NNI and $1.3 \mathrm{~cm}^{-1}$ in para-NNI-EDGs. Although all these values are within the error bar our methodology for $V_{S O C}$ calculation, ${ }^{35}$ we can speculate that the slightly larger value upon meta-substitutions are due to the asymmetry it introduces, helping to mix the singly-occupied orbitals in the singlet and triplet states.

The fluorescence rates are also more significantly impacted by the meta- than by the para-substitutions. While in the latter, they are reduced by a factor 35 compared to NNI, in upon a meta-substitution this reduction is between 64 and 77 times. Thus, the fluorescence lifetime is elongated up to $408 \mathrm{~ns}$. Once more, the reduction of the fluorescence rates is due to the dark nature of $S_{1}$.

Efficient ISC is the prerequisite for photoinduced phosphorescence process. It is crucial to enhance ISC in molecular design towards organic phosphorescence. ${ }^{14}$ In this theoretical study, we showed that for specific fluorescent molecules like NNI, 
chemical modification by adding an electron donating group (EDG) could appreciably shift the balance between fluorescence and ISC, favoring the latter. In NNI and NNI with electron withdrawing group (EWG), ISC occurs within $\sim 25 \mathrm{~ns}$, while fluorescence is faster, occurring within $5 \mathrm{~ns}$. Thus, these molecules tend to show strong fluorescence. In NNI-EDG, however, ISC becomes faster, between 3 and $11 \mathrm{ns,}$, while fluorescence becomes much longer, between 185 and 408 ns. Therefore, these molecules efficiently populate the triplet manifold and, under adequate conditions, may become phosphorescent. It is encouraging to see that the EDG substitution enhances the transference from singlet to triplet states and enables phosphorescence, even in systems with very small spin-orbit couplings $\left(\sim 1 \mathrm{~cm}^{-1}\right)$, as it is often the case of pure light-atoms organic molecules.

The strong photophysical shift between NNI and NNI-EWG on the one hand and NNI-EDG on the other is related to the nature of the $S_{1}$ state. In the former molecules, the lowest singlet state has a localized character, while upon an EDG substitution it becomes a charge-transfer (CT) state in response to the configurational change of molecules. The $\mathrm{CT}$ state has a slightly stronger coupling to $\mathrm{T}_{2}$. Although the spin-orbit coupling is still tiny, about $2 \mathrm{~cm}^{-1}$, it is enough to enhance the ISC rate by up to a factor 7 compared to the parent NNI. Concerning fluorescence, while the localized $S_{1}$ state is strongly dipole-coupled to $S_{0}$, with oscillator strength near 0.4 , the CT $S_{1}$ state has a vanishing coupling to $\mathrm{S}_{0}$, with an oscillator strength not larger than 0.01 . Therefore, the fluorescence rate reduces by up 77 times.

These are systematic findings observed in the comparison between the parent NNI and NNI functionalized by $-\mathrm{F}$ and $-\mathrm{COOH}$ EWGs and $-\mathrm{OCH}_{3}$ and $-\mathrm{OH}$ EDGs. Substitution of these groups in either para or meta locations does not change the qualitative results, although the population of triplet states is even more favorable after a meta-substitution.

In short, we have shown that ISC can be promoted by restrained fluorescence due to low-lying CT singlet states. The triplet population formed in this way may 
fluoresce as long as conventional conditions are met: nonradiative rates are small, and the chromophore is in an oxygen-depleted atmosphere. Restrained-fluorescence ISC turns out to be so efficient that it can deplete the singlet population even in systems with spin-orbit couplings as small as those in El-Sayed forbidden transitions.

This study cast new insight into the design of pure light-atoms organic phosphorescent molecules via chemical modifications of functional groups. By exploring more organic molecules using the same strategy and employing more comprehensive theoretical framework that can compute phosphorescent radiative decay rate ${ }^{44-45}$ we are on the way of rational design of new organic phosphorescent molecules.

\section{AUTHOR INFORMATION}

\section{Corresponding Author}

*(G.Z.) E-mail: guozhen@ustc.edu.cn.

*(M.B.) E-mail: mario.barbatti@univ-amu.fr.

\section{ORCID}

Guozhen Zhang: 0000-0003-0125-9666

Mario Barbatti: 0000-0001-9336-6607

\section{Note}

The authors declare no competing financial support.

\section{Acknowledgments}

This work was financially supported by MOST (No. 2016YFA0400904), NSFC (21703221, 21633006), and the Fundamental Research Funds for the Central Universities (WK2060030027). RL and GZ are grateful for the computing resources from University of Science and Technology of China High Performance Computing Center. MB thanks the support of the Excellence Initiative of Aix-Marseille University 
(A*MIDEX) and the project Equip@ Meso (ANR-10-EQPX-29-01), both funded by the French Government "Investissements d'Avenir" program. MB also acknowledges funding from the WSPLIT project (ANR-17-CE05-0005-01).

\section{Supporting Information Avaliable}

The computational details, transition energies, transition related molecular orbitals and corresponding electron-hole distribution of the lowest singlet excited state, the energy level diagrams of low-lying excited states, the Cartesian coordinates of all optimized molecules.

\section{References}

1. He, Z.; Zhao, W.; Lam, J. W. Y.; Peng, Q.; Ma, H.; Liang, G.; Shuai, Z.; Tang, B. Z. White Light Emission from a Single Organic Molecule with Dual Phosphorescence at Room Temperature. Nat Commun 2017, 8, 416.

2. Zhao, W.; He, Z.; Lam, Jacky W. Y.; Peng, Q.; Ma, H.; Shuai, Z.; Bai, G.; Hao, J.; Tang, Ben Z. Rational Molecular Design for Achieving Persistent and Efficient Pure Organic RoomTemperature Phosphorescence. Chem 2016, 1, 592-602.

3. Chen, X., et al. Versatile Room-Temperature-Phosphorescent Materials Prepared from NSubstituted Naphthalimides: Emission Enhancement and Chemical Conjugation. Angew Chem Int Ed Engl 2016, 55, 9872-6.

4. Lee, J.; Chen, H. F.; Batagoda, T.; Coburn, C.; Djurovich, P. I.; Thompson, M. E.; Forrest, S. R. Deep Blue Phosphorescent Organic Light-Emitting Diodes with Very High Brightness and Efficiency. Nat Mater 2016, 15, 92-8.

5. Zhen, X.; Tao, Y.; An, Z.; Chen, P.; Xu, C.; Chen, R.; Huang, W.; Pu, K. Ultralong Phosphorescence of Water-Soluble Organic Nanoparticles for in Vivo Afterglow Imaging. $A d v$. Mater. 2017, 29.

6. Baryshnikov, G.; Minaev, B.; Agren, H. Theory and Calculation of the Phosphorescence Phenomenon. Chem. Rev. 2017, 117, 6500-6537. 
7. Kwon, M. S., et al. Suppressing Molecular Motions for Enhanced Room-Temperature Phosphorescence of Metal-Free Organic Materials. Nat Commun 2015, 6, 8947.

8. Hedley, G. J.; Ruseckas, A.; Samuel, I. D. Ultrafast Intersystem Crossing in a Red Phosphorescent Iridium Complex. J. Phys. Chem. A 2009, 113, 2-4.

9. Rothe, C.; King, S.; Monkman, A. Long-Range Resonantly Enhanced Triplet Formation in Luminescent Polymers Doped with Iridium Complexes. Nat. Mater. 2006, 5, 463-6.

10. Acharya, R.; Cekli, S.; Zeman, C. J. t.; Altamimi, R. M.; Schanze, K. S. Effect of Selenium Substitution on Intersystem Crossing in Pi-Conjugated Donor-Acceptor-Donor Chromophores: The LUMO Matters the Most. J. Phys. Chem. Lett. 2016, 7, 693-7.

11. Samanta, P. K.; Kim, D.; Coropceanu, V.; Bredas, J. L. Up-Conversion Intersystem Crossing Rates in Organic Emitters for Thermally Activated Delayed Fluorescence: Impact of the Nature of Singlet Vs Triplet Excited States. J. Am. Chem. Soc. 2017, 139, 4042-4051.

12. Lucenti, E.; Forni, A.; Botta, C.; Carlucci, L.; Giannini, C.; Marinotto, D.; Previtali, A.; Righetto, S.; Cariati, E. H-Aggregates Granting Crystallization-Induced Emissive Behavior and Ultralong Phosphorescence from a Pure Organic Molecule. J. Phys. Chem. Lett. 2017, 8, 18941898.

13. Bai, F. Q.; Nakatani, N.; Nakayama, A.; Hasegawa, J. Y. Excited States of a Significantly Ruffled Porphyrin: Computational Study on Structure-Induced Rapid Decay Mechanism Via Intersystem Crossing. J. Phys. Chem. A 2014, 118, 4184-94.

14. Ma, H.; Peng, Q.; An, Z.; Huang, W.; Shuai, Z. Efficient and Long-Lived RoomTemperature Organic Phosphorescence: Theoretical Descriptors for Molecular Designs. J. Am. Chem. Soc. 2019.

15. Vlček, A.; Záliš, S. Modeling of Charge-Transfer Transitions and Excited States in D6 Transition Metal Complexes by Dft Techniques. Coord. Chem. Rev. 2007, 251, 258-287.

16. Thomas, R. A.; Tsai, C. N.; Mazumder, S.; Lu, I. C.; Lord, R. L.; Schlegel, H. B.; Chen, Y. J.; Endicott, J. F. Energy Dependence of the Ruthenium(Ii)-Bipyridine Metal-to-LigandCharge-Transfer Excited State Radiative Lifetimes: Effects of Pipi*(Bipyridine) Mixing. $J$. Phys. Chem. B 2015, 119, 7393-406. 
17. Xu, H.; Chen, R.; Sun, Q.; Lai, W.; Su, Q.; Huang, W.; Liu, X. Recent Progress in MetalOrganic Complexes for Optoelectronic Applications. Chem. Soc. Rev. 2014, 43, 3259-302.

18. Mukherjee, S.; Thilagar, P. Recent Advances in Purely Organic Phosphorescent Materials. Chem Commun (Camb) 2015, 51, 10988-1003.

19. McClenaghan, N. D.; Leydet, Y.; Maubert, B.; Indelli, M. T.; Campagna, S. Excited-State Equilibration: A Process Leading to Long-Lived Metal-to-Ligand Charge Transfer Luminescence in Supramolecular Systems. Coord. Chem. Rev. 2005, 249, 1336-1350.

20. Yushchenko, O.; Licari, G.; Mosquera-Vazquez, S.; Sakai, N.; Matile, S.; Vauthey, E. Ultrafast Intersystem-Crossing Dynamics and Breakdown of the Kasha-Vavilov's Rule of Naphthalenediimides. J. Phys. Chem. Lett. 2015, 6, 2096-100.

21. Joo, B.; Kim, E. G. Model-Independent Determination of the Degree of Charge Transfer in Molecular and Metal Complexes. Chem. Commun. 2015, 51, 15071-4.

22. Niehaus, T. A.; Hofbeck, T.; Yersin, H. Charge-Transfer Excited States in Phosphorescent Organo-Transition Metal Compounds: A Difficult Case for Time Dependent Density Functional Theory? RSC Advances 2015, 5, 63318-63329.

23. Han, J.; Shen, L.; Chen, X.; Fang, W. Phosphorescent Mechanism for Single-Dopant White Oled of Fpt: Electronic Structure and Electron Exchange-Induced Energy Transfer. Journal of Materials Chemistry C 2013, 1, 4227.

24. Yang, Z.; Mao, Z.; Xie, Z.; Zhang, Y.; Liu, S.; Zhao, J.; Xu, J.; Chi, Z.; Aldred, M. P. Recent Advances in Organic Thermally Activated Delayed Fluorescence Materials. Chem. Soc. Rev. 2017, 46, 915-1016.

25. Yang, L.; Wang, X.; Zhang, G.; Chen, X.; Zhang, G.; Jiang, J. Aggregation-Induced Intersystem Crossing: A Novel Strategy for Efficient Molecular Phosphorescence. Nanoscale 2016, $8,17422-17426$.

26. Dias, F. B.; Bourdakos, K. N.; Jankus, V.; Moss, K. C.; Kamtekar, K. T.; Bhalla, V.; Santos, J.; Bryce, M. R.; Monkman, A. P. Triplet Harvesting with 100\% Efficiency by Way of Thermally Activated Delayed Fluorescence in Charge Transfer Oled Emitters. Adv. Mater. 2013, 25, 3707-14. 
27. Endo, A.; Sato, K.; Yoshimura, K.; Kai, T.; Kawada, A.; Miyazaki, H.; Adachi, C. Efficient up-Conversion of Triplet Excitons into a Singlet State and Its Application for Organic Light Emitting Diodes. Appl. Phys. Lett. 2011, 98, 083302.

28. Chabera, P., et al. A Low-Spin Fe(Iii) Complex with 100-Ps Ligand-to-Metal Charge Transfer Photoluminescence. Nature 2017, 543, 695-699.

29. Bolton, O.; Lee, K.; Kim, H. J.; Lin, K. Y.; Kim, J. Activating Efficient Phosphorescence from Purely Organic Materials by Crystal Design. Nat Chem. 2011, 3, 205-10.

30. An, Z., et al. Stabilizing Triplet Excited States for Ultralong Organic Phosphorescence. Nat Mater 2015, 14, 685-90.

31. Qian, Y.; Li, S.; Zhang, G.; Wang, Q.; Wang, S.; Xu, H.; Li, C.; Li, Y.; Yang, G. Aggregation-Induced Emission Enhancement of 2-(2'-Hydroxyphenyl)Benzothiazole-Based Excited-State Intramolecular Proton-Transfer Compounds. J. Phys. Chem. B 2007, 111, 58618.

32. Ravotto, L.; Ceroni, P. Aggregation Induced Phosphorescence of Metal Complexes: From Principles to Applications. Coord. Chem. Rev. 2017, 346, 62-76.

33. Mei, J.; Leung, N. L.; Kwok, R. T.; Lam, J. W.; Tang, B. Z. Aggregation-Induced Emission: Together We Shine, United We Soar! Chem. Rev. 2015, 115, 11718-940.

34. El-Sayed, M. A. Spin-Orbit Coupling and the Radiationless Processes in Nitrogen Heterocyclics. J. Chem. Phys. 1963, 38, 2834-2838.

35. Gao, X.; Bai, S.; Fazzi, D.; Niehaus, T.; Barbatti, M.; Thiel, W. Evaluation of Spin-Orbit Couplings with Linear-Response Time-Dependent Density Functional Methods. J. Chem. Theory Comput. 2017, 13, 515-524.

36. Dinkelbach, F.; Kleinschmidt, M.; Marian, C. M. Assessment of Interstate Spin-Orbit Couplings from Linear Response Amplitudes. J. Chem. Theory Comput. 2017, 13, 749-766.

37. Sayfutyarova, E. R.; Chan, G. K.-L. A State Interaction Spin-Orbit Coupling Density Matrix Renormalization Group Method. J. Chem. Phys. 2016, 144, 234301.

38. Bredas, J. L.; Beljonne, D.; Coropceanu, V.; Cornil, J. Charge-Transfer and EnergyTransfer Processes in Pi-Conjugated Oligomers and Polymers: A Molecular Picture. Chem. Rev. 2004, 104, 4971-5004. 
39. Chiodo, S. G.; Russo, N. One-Electron Spin-Orbit Contribution by Effective Nuclear Charges. J. Comput. Chem. 2009, 30, 832-839.

40. Casida, M. E. Time-Dependent Density Functional Response Theory for Molecules. In Recent Advances in Density Functional Methods, Chong, D. P., Ed. World Scientific: Singapore, 1995; Vol. 1, pp 155-192.

41. Hilborn, R. C. Einstein Coefficients, Cross-Sections, F Values, Dipole-Moments, and All That. Am. J. Phys. 1982, 50, 982-986.

42. Gong, Z. Y.; Duan, S.; Tian, G.; Zhang, G.; Jiang, J.; Luo, Y. Optical Excitation in DonorPt-Acceptor Complexes: Role of the Structure. J. Phys. Chem. A 2016, 120, 3547-53.

43. Marian, C. M. Spin-Orbit Coupling and Intersystem Crossing in Molecules. WIREs: Comp. Mol. Sci. 2012, 2, 187-203.

44. Peng, Q.; Niu, Y.; Shi, Q.; Gao, X.; Shuai, Z. Correlation Function Formalism for Triplet Excited State Decay: Combined Spin-Orbit and Nonadiabatic Couplings. J. Chem. Theory Comput. 2013, 9, 1132-43.

45. Minaev, B.; Baryshnikov, G.; Agren, H. Principles of Phosphorescent Organic Light Emitting Devices. Phys. Chem. Chem. Phys. 2014, 16, 1719-1758. 